\title{
Detecting pre-death grief in family caregivers of persons with dementia: measurement equivalence of the Mandarin- Chinese version of Marwit-Meuser caregiver grief inventory
}

Tau Ming Liew ${ }^{1,2,3^{*}}$, Philip Yap ${ }^{4,5}$, Nan Luo ${ }^{3}$, Soo Boon Hia', Gerald Choon-Huat Koh ${ }^{3}$ and Bee Choo Tai ${ }^{3}$

\begin{abstract}
Background: Pre-death grief (PDG) is a key challenge faced by caregivers of persons with dementia (PWD). MarwitMeuser Caregiver Grief Inventory (MM-CGI) and its abbreviated MM-CGI-Short-Form (MM-CGI-SF) are among the few empirically-developed scales that detect PDG. However, they have not had a Mandarin-Chinese version even though Chinese-speaking populations have among the largest number of PWD. We produced a Mandarin-Chinese version of MM-CGI and evaluated whether it had equivalent scores and similar psychometric properties to the English version.
\end{abstract}

Methods: We produced the Chinese MM-CGI through the methods of forward-backward translation and cognitive debriefing. Then, we recruited family caregivers of PWD $(n=394)$ to complete either the Chinese $(n=103)$ or English $(n=291)$ version. The two versions were compared in their score-difference (adjusting for potential confounders using multiple linear regression), internal-consistency reliability (using Cronbach's a) and testretest reliability (using intraclass correlation-coefficient), known-group validity (based on the relationship with the PWD and stage of dementia) and construct validity (using Spearman's correlation-coefficient).

Results: The two versions showed similar mean scores, with the adjusted score-difference of $1.2(90 \% \mathrm{Cl}-5.6$ to 7.9$)$ for MM-CGl and- $0.4(90 \% \mathrm{Cl}-2.9$ to 2.1) for MM-CGI-SF. The $90 \% \mathrm{Cl}$ for adjusted score-difference fell within predefined equivalence-margin ( \pm 8 for MM-CGI and \pm 3 for MM-CGI-SF) and indicated equivalence of the scores. The two versions also demonstrated similar characteristics in reliability and validity.

Conclusions: The Chinese MM-CGI opens the way for PDG assessment and intervention among Chinese-speaking caregivers. Establishing its measurement equivalence with the English version paves the way for cross-cultural research on PDG in dementia caregiving.

Keywords: Pre-death grief, Family caregivers, Dementia, Chinese, Marwit-Meuser caregiver grief inventory, Measurement equivalence

\footnotetext{
* Correspondence: tau_ming_liew@imh.com.sg

${ }^{1}$ Department of Geriatric Psychiatry, Institute of Mental Health, 10 Buangkok View, Singapore, Singapore

${ }^{2}$ Psychotherapy Service, Institute of Mental Health, Singapore, Singapore

Full list of author information is available at the end of the article
}

(c) The Author(s). 2018 Open Access This article is distributed under the terms of the Creative Commons Attribution 4.0 International License (http://creativecommons.org/licenses/by/4.0/), which permits unrestricted use, distribution, and reproduction in any medium, provided you give appropriate credit to the original author(s) and the source, provide a link to the Creative Commons license, and indicate if changes were made. The Creative Commons Public Domain Dedication waiver (http://creativecommons.org/publicdomain/zero/1.0/) applies to the data made available in this article, unless otherwise stated. 


\section{Background}

With the rising prevalence of dementia globally [1], more family members will inevitably be drawn into the care of their relatives with dementia. Providing care to a person with dementia (PWD) is often protracted, stressful [2] and not uncommonly associated with physical and emotional burden [2-4]. Caregiver burden, in turn, has been shown to produce less desirable outcomes such as premature nursing home placement of the PWD [5] and greater risk of mortality in the PWD [6]. For this reason, much research has targeted caregiver burden and the appropriate interventions to alleviate burden [3]. However, the loss and grief that caregivers often experience while providing care is less commonly recognised.

Caregivers of PWD may begin their bereavement long before the physical death of the PWD [7]. The caregivers' responses to perceived losses in the pre-death context - which we refer to as pre-death grief (PDG) include the anticipation of future loss related to the physical death of PWD and the mourning of present loss related to the psychological death of PWD (a unique phenomenon in dementia whereby the PWD is still physically present but emotionally disconnected from the caregiver) [8]. In a recent systematic review from the British Journal of Psychiatry [9], PDG has been highlighted as a key challenge faced by caregivers, with the loss of emotional bond with PWD accentuating caregivers' unmet needs for nurture, companionship and emotional security. PDG has also been described as the central experience of spousal caregivers of PWD in another recent systematic review of the qualitative studies [10]. The prevalence of PDG has been reported in a few studies and ranged between 47 and $86 \%[8,11]$.

PDG in dementia caregiving can be more complicated than the usual experience of loss and grief $[8,12]$. The protracted trajectory of dementia prolongs the caregivers' sense of uncertainty about the loss. During this protracted period of uncertainty, the communication with the PWD also becomes disrupted and there is no longer any opportunity to reaffirm the relationship. To make the matter worse, such experience of grief before the physical death of PWD may not be socially sanctioned, leaving the caregivers with the sense of isolation as they find it difficult to share their feelings with the people around them. When the experience of loss and grief is not recognized, caregivers may have difficulty accepting any changes that have occurred in the PWD and may attempt to fight the inevitable decline in the PWD. To that end, they may be more inclined to cope using control-based strategies, become more paternalistic in their communication with the PWD and adopt more authoritarian decision-making styles in caregiving. Eventually, this can affect the well-being of both the
PWD and the caregivers, with the PWD feeling the sense of loss of autonomy and the caregivers feeling more helpless over what they cannot control [13]. Not surprisingly, high levels of PDG have been associated with caregiver burden [14] and depression [11], and increase the risk of caregivers considering nursing home placement for the PWD [15]. However, caregivers with high PDG may not be easily recognised in clinical practice because PDG is commonly disenfranchised [12] and not readily talked about by caregivers. They may also be missed by other routinely-used scales such as the caregiver burden scale as demonstrated by a recent study [8], thus indicating the need for a scale that specifically measures PDG.

The Marwit-Meuser Caregiver Grief Inventory (MMCGI) [16] and its abbreviated MM-CGI-Short-Form (MM-CGI-SF) [17] are among the few empiricallydeveloped scales that measure PDG [11, 12]. However, it has not had a Mandarin-Chinese version even though the Mandarin-Chinese language is among the most widely-used language worldwide [18], and countries with Chinese-speaking populations have among the largest number of PWD (with 9.5 million in China alone) [1]. Although there was a recent study that validated the Chinese MM-CGI-SF, it only focused on the CantoneseChinese version [19]. Cantonese-Chinese is a dialect of the Chinese language that is used in only $5 \%$ of the Chinese-speaking community [18], in contrast to the Mandarin-Chinese which is used in $70 \%$ of the community [18] and is recognised as the national language in countries such as China and Taiwan. While the Cantonese-Chinese and Mandarin-Chinese share similarities in the written characters, they are distinct and may not be mutually intelligible.

In this study, we produced the Mandarin-Chinese version of MM-CGI for use among Chinese-speaking caregivers. As the language of a scale can have prominent influence [20] on individuals' responses to culturally-sensitive questions (such as those related to grief) [21], we are uncertain whether the new Mandarin-Chinese version will be equivalent in its measurement scores to those of the original English version. This is an important issue for future research that endeavours to compare the scores between the Chineseand English-speaking populations in cross-cultural studies, or pool data from the two language versions to improve statistical power and representativeness of a study. As such, we sought to evaluate whether the Chinese versions of MM-CGI and its abbreviated MM-CGI-SF are equivalent in their measurement scores to the original English version. As a secondary aim, we also sought to evaluate whether the Chinese and English versions demonstrate similar patterns in reliability and validity assessment. 


\section{Methods}

\section{Development of the Chinese version of Marwit-Meuser} caregiver grief inventory

We produced the Mandarin-Chinese version of MMCGI [16] by applying the methods which have been recommended by the International Society for Pharmacoeconomics and Outcomes Research (ISPOR) [22]. Briefly, the methods included forward translation, backtranslation and cognitive debriefing. The details of each step are described in the paragraphs below.

In the first step (forward translation), two native speakers of Chinese language (SJCL and SBH) independently translated the MM-CGI into Chinese versions. Both copies of the forward translation were reconciled by an independent native speaker of Chinese language (TML), in discussion with the two forward translators whenever necessary.

In the second step (back-translation), two other persons translated the reconciled Chinese version back to the English language (HLS and DLLC). These two backtranslators were native speakers of English language who had not seen the original MM-CGI. Thereafter, two independent reviewers (KST and TML) compared the back-translations against the original English version of MM-CGI to resolve any discrepancies.

The final step, cognitive debriefing, involves conducting qualitative interviews on a small group of people from the target language to assess the level of comprehensibility and cognitive equivalence of the translation, and to identify any items that may potentially cause confusion [22]. We conducted the cognitive debriefings on three professional caregivers and three family caregivers - the three professional caregivers (YH, HL and CL) were nurses who were working in a geriatric psychiatry ward and had at least three years of experience in the routine care of PWD; while the three family caregivers were children caregivers who accompanied their relatives with dementia for routine clinical care. During the cognitive debriefing, we asked the caregivers to rephrase each item in MM-CGI to assess their understanding. We also encouraged caregivers to highlight any items that were difficult to understand. Then, two researchers (BIY and TML) independently reviewed the transcripts of the verbatim responses. They proposed amendments when both of them agreed that an item was unclear or was interpreted differently from the original intent. Any disagreement was resolved by referring to a third researcher (PY). Any proposed amendment also required the review and agreement by the third researcher (PY).

The final version of Chinese MM-CGI is presented in Additional file 1.

\section{Participants and procedures}

Upon producing the Chinese MM-CGI, we consecutivelysampled caregivers from two tertiary hospitals in the
North-East of Singapore to complete the Chinese version of MM-CGI. These are caregivers who accompanied the PWD to the dementia care services and fulfilled the following criteria: (i) spouses or children of PWD; (ii) caring for PWD who is residing in the community; (iii) age $\geq 21$ years; and (iv) able to read in Chinese. The recruitment criteria were predicated on our definition of family caregivers, which includes family members who are involved in the care of the PWD either directly or indirectly [23] - hence, we considered the act of accompanying the PWD to the dementia care services as an evidence of indirect involvement with the care of the PWD, which qualifies a person as a family caregiver. At the point of recruitment, the participants completed on-site the Chinese version of MM-CGI, together with a caregiver burden scale (Zarit Burden Interview, ZBI) and a depression scale (Centre for Epidemiologic Studies-Depression Scale, CES-D). One week later, the participants were reminded through phone to complete a second questionnaire at home and to mail back the completed questionnaire to us, for the purpose of assessing test-retest reliability.

We also needed participants who have completed the English MM-CGI to allow comparisons between the Chinese and English versions of MM-CGI. To that end, we included the dataset from our separate study [8] which recruited 300 English-speaking caregivers to complete the English MM-CGI. This other study was a replica of the current study (sharing the same recruitment site, study period, inclusion criteria and study procedures), with the only difference in the language of administration (the separate study was in English, in contrast to the current study which was in Chinese). The details of this other study are also available at [8].

Altogether, we recruited 403 participants, with 103 completing the Chinese version and 300 (from our separate study) [8] completing the English version. We limited our analysis to one caregiver for each PWD in the study to avoid between-participant correlation among those from the same household and consequently, excluded 9 participants from the English version because they were from the same household as some of those who completed the Chinese version. Hence, the final analysis only included 394 participants (103 in the Chinese version and 291 in the English version), with one caregiver for each PWD in the study. Following the initial questionnaire, $60 \%$ of the participants mailed back a second set of the questionnaire for assessment of test-retest reliability (61.2\% among those who completed the Chinese version and $59.8 \%$ among the English version). Median time of completing the second questionnaire was 11 days, with an inter-quartile range of 8-13. 
The Domain Specific Review Board of Singapore granted ethical approval for the study.

\section{Measures}

The Marwit-Meuser Caregiver Grief Inventory (MMCGI) is among the few PDG scales that has been empirically-developed, following extensive focus-group interviews with caregivers of PWD [16]. It has 50 items that assess PDG on 5-point Likert scales and produces a maximum score of 250 . The original validation study [16] also revealed 3 dimensions of loss in the exploratory factor analysis - Personal Sacrifice Burden, Heartfelt Sadness and Longing, and Worry and Felt Isolation. The Personal Sacrifice Burden dimension captures the personal aspect of losses experienced by caregivers (such as loss of personal freedom, sleep and physical health), the Heartfelt Sadness and Longing dimension captures the traditional concept of grief (that is, one's intrapersonal reactions to lost relationship) and the Worry and Felt Isolation dimension captures the feelings of losing connection with others and the worry about future losses. These 3 dimensions of loss are not dissimilar to the 3 aspects of loss previously conceptualized by Dempsey and Baago [24], namely the loss of personal identity, loss of the PWD, and symbolic loss of the ideal. MM-CGI also has a shorter version, the MM-CGI-Short Form (MMCGI-SF), which uses only the 18 most representative items for ease of administration [17]. MM-CGI-SF is also rated on 5-point Likert scales and has a maximum score of 90. Both MM-CGI and MM-CGI-SF have been validated in Singapore [8].

The Zarit Burden Interview (ZBI) is a 22-item scale that measures the perceived burden experienced by caregivers of older persons. It is scored on 5-point Likert scales, with the total score ranging from 0 to 88 . According to the original test instructions [25], a score range of 21-40 indicates mild burden, while 41-60 indicates moderate burden and 61-88 indicates high burden. In a more recent study [26], scores $\geq 34$ were reported to indicate significant burden that may require clinical attention. ZBI has been shown to contain 5 domains - Burden in the Relationship, Emotional Well-being, Social and Family Life, Finances, and Loss of Control over one's life [27]. The Centre for Epidemiologic Studies-Depression Scale (CES-D) comprises 20 items which measure depressive symptomatology on 4-point Likert scales. The total score ranges from 0 to 60 , with scores $\geq 16$ indicating significant depression $[28,29]$. CES-D contains 4 domains - Depressed affect, Positive Affect, Somatic Symptoms, and Interpersonal Problems. ZBI and CES-D have previously been validated in Singapore [30, 31].

Key information on the caregiver and PWD was also captured in the study. The information was based on self-reports by the caregivers or obtained from the medical records when the caregivers were uncertain. Caregiver data included age, gender, ethnicity, marital status, employment status, educational attainment, relationship with PWD, whether the caregiver stayed with the PWD, duration of caregiving, frequency of contact with the PWD and primary caregiving role. Data relating to the PWD included age, gender, duration of dementia diagnosis and stage of dementia. The stage of dementia was captured using a brief measure based on the descriptions of the three dementia severities described in the revised third edition of Diagnostic and Statistical Manual of Mental Disorders (DSM-III-R) [32]. From the three options, participants chose the description that best described the PWD - still capable of independent living (mild stage), needs some assistance with daily living (moderate stage), or needs round-the-clock supervision (severe stage). This brief measure was previously shown to have reasonable agreement (kappa 0.56-0.6) [33, 34] with Clinical Dementia Rating Scale, which is one of the most commonly-used scale to stage dementia $[35,36]$. This brief measure is also nearly identical to the re-introduced dementia severity in DSM-5 [37].

\section{Statistical analyses}

We computed the score-difference between the language versions using multiple linear regression, adjusting for potential confounding variables (relationship with PWD, stage of dementia and the baseline variables which were significantly different between the two language versions). Relationship with the PWD and stage of dementia were included in the statistical adjustment because these two variables have been reported in the literature to be predictors of PDG [11, 38].

Based on the approach to evaluate therapeutic equivalence in clinical trials, we defined a priori that equivalence in the scores of the Chinese and English versions would be declared if the $90 \%$ confidence interval (CI) of the adjusted score-difference fell within a predefined margin of equivalence. The use of $90 \% \mathrm{CI}$ is in line with internationally-accepted practice of evaluating bioequivalence in clinical trials $[39,40]$, and corresponds to conducting two one-sided tests of hypothesis at the $5 \%$ level of significance [41]. The margin of equivalence refers to a predefined range of values that would be considered too small to be clinically meaningful, and any differences which are lesser than these predefined values would be small enough to reasonably claim equivalence. In this study, we predefined the margin of equivalence as approximately $\pm 5 \%$ of the reported average values of MM-CGI and MM-CGI-SF from the original publications $[16,17]$. This translated into margins of equivalence of \pm 8 for MM-CGI and \pm 3 for MM-CGI-SF. The respective margins of equivalence for the subscales of MM-CGI and MM-CGI-SF are also presented in Table 2. 
Table 1 Demographic information of the caregivers and the persons with dementia $(n=394)$

\begin{tabular}{|c|c|c|c|c|}
\hline Variable & Overall sample $(n=394)$ & Chinese version $(n=103)$ & English version $(n=291)$ & $P$ value \\
\hline \multicolumn{5}{|l|}{ Variables related to caregivers } \\
\hline Age, mean (SD) & $53.0(10.7)$ & $55.2(9.3)$ & $52.2(11.0)$ & 0.015 \\
\hline Female gender, $n(\%)$ & $236(59.9)$ & $61(59.2)$ & $175(60.1)$ & 0.871 \\
\hline Ethnic, $n(\%)$ & & & & $<0.001$ \\
\hline Chinese & $341(86.6)$ & $103(100)$ & $238(81.8)$ & \\
\hline Malay & $25(6.3)$ & $0(0)$ & $25(8.6)$ & \\
\hline Indian & $18(4.6)$ & $0(0)$ & $18(6.2)$ & \\
\hline Others & $10(2.5)$ & $0(0)$ & $10(3.4)$ & \\
\hline Marital status, $n(\%)$ & & & & 0.630 \\
\hline Married & $271(68.8)$ & $74(71.8)$ & $197(67.7)$ & \\
\hline Single & $94(23.9)$ & $21(20.4)$ & $73(25.1)$ & \\
\hline Widowed/Divorced/Separated & $29(7.3)$ & $8(7.8)$ & $21(7.2)$ & \\
\hline Employment status, $n$ (\%) & & & & 0.059 \\
\hline Not working & $123(31.2)$ & $40(38.8)$ & $83(28.5)$ & \\
\hline Working part-time & $52(13.2)$ & $16(15.5)$ & $36(12.4)$ & \\
\hline Working full-time & $219(55.6)$ & $47(45.6)$ & $172(59.1)$ & \\
\hline Highest education, $n$ (\%) & & & & $<0.001$ \\
\hline Primary or no formal education & $41(10.4)$ & $33(32.0)$ & $8(2.8)$ & \\
\hline Secondary & $228(57.9)$ & $54(52.4)$ & $174(59.8)$ & \\
\hline Tertiary & $125(31.7)$ & $16(15.5)$ & $108(37.5)$ & \\
\hline Relationship with PWD, n (\%) & & & & 0.104 \\
\hline Child & $340(86.3)$ & $84(81.6)$ & $256(88.0)$ & \\
\hline Spouse & $54(13.7)$ & 19 (18.5) & $35(12.0)$ & \\
\hline Staying with PWD, n (\%) & $264(67.0)$ & $70(68.0)$ & $194(66.7)$ & 0.810 \\
\hline Duration of caregiving in years, mean (SD) & $6.8(6.7)$ & $7.2(6.6)$ & $6.6(6.7)$ & 0.514 \\
\hline Frequency of contact with PWD, $n(\%)$ & & & & 0.436 \\
\hline Daily, for at least $4 \mathrm{~h}$ a day & $211(53.6)$ & $59(57.3)$ & $152(52.2)$ & \\
\hline Daily, but less than $4 \mathrm{~h}$ a day & $79(20.0)$ & $15(14.6)$ & $64(22.0)$ & \\
\hline At least once a week & $84(21.3)$ & $24(23.3)$ & $60(20.6)$ & \\
\hline Less than once a week & $20(5.1)$ & $5(4.9)$ & $15(5.2)$ & \\
\hline Primary caregiving role, $n$ (\%) & $279(70.8)$ & $65(63.1)$ & $214(73.5)$ & 0.045 \\
\hline ZBI score, mean (SD) & $34.8(16.8)$ & $33.5(14.1)$ & $35.3(17.6)$ & 0.331 \\
\hline CES-D score, mean (SD) & $15.7(11.0)$ & $17.4(11.5)$ & $15.1(10.8)$ & 0.064 \\
\hline \multicolumn{5}{|l|}{ Variables related to PWD } \\
\hline Age, mean (SD) & $79.5(8.2)$ & $79.7(8.3)$ & $79.4(8.1)$ & 0.779 \\
\hline Female gender, $n(\%)$ & $278(70.6)$ & $72(69.9)$ & $206(70.8)$ & 0.865 \\
\hline Duration of dementia diagnosis in years, mean (SD) & $4.5(3.5)$ & $4.3(3.3)$ & $4.5(3.5)$ & 0.500 \\
\hline Stage of dementia, $n$ (\%) & & & & 0.626 \\
\hline Mild & $62(15.7)$ & $14(13.6)$ & $48(16.5)$ & \\
\hline Moderate & $163(41.4)$ & $41(39.8)$ & $122(41.9)$ & \\
\hline Severe & 169(42.9) & 48 (46.6) & 121 (41.6) & \\
\hline
\end{tabular}

SD, standard deviation; PWD, persons with dementia; ZBI, Zarit Burden Interview; CES-D; Centre for Epidemiologic Studies Depression Scale ${ }^{\text {aT }}$ Test of difference between Chinese and English versions: chi-square test for categorical variables and two-sample T-test for continuous variables. Bold-faced $p$ values are $\leq 0.05$ 
Should the $90 \%$ CI fall outside of the $\pm 5 \%$ margin, we will still consider the presence of possible equivalence if the confidence interval does not exceed the lessstringent $\pm 10 \%$ margin (which is \pm 16 for MM-CGI and \pm 6 for MM-CGI-SF). On the other hand, should the $90 \%$ CI fall entirely outside of the $10 \%$ margin, we will declare that there are significant differences in the scores between the Chinese and English versions.

Additionally, we also compared the two language versions with respect to their reliability and validity - we expected that the $95 \% \mathrm{CI}$ of all the reliability indices should fall within the range of $\geq 0.70$ (a value indicating the minimally-acceptable reliability) $[42,43]$ and the validity assessment should yield similar patterns between the two languages. We evaluated the internal-consistency reliability with Cronbach's $\alpha$; test-retest reliability with intraclass correlation coefficient (ICC); known-group validity by comparing mean scores based on the relationship with the PWD and the stage of dementia (these two variables have been known to demonstrate contrasting levels of PDG in the literature) [8, 10, 11,38]; and construct validity by correlation with ZBI and CES-D using Spearman's correlation-coefficient $(\rho)$. All analyses were performed with STATA software version 13.

\section{Results}

The demographic information of the 394 participants is shown in Table 1. The participants comprised multiple ethnicities, with a predominance of Chinese (86.6\%). Spousal caregivers constituted $13.7 \%$, with the rest being children caregivers. Participants who completed the Chinese version were slightly older $(p=0.015)$, less educated $(p<0.001)$ and less likely to be primary caregivers $(p=0.045)$.

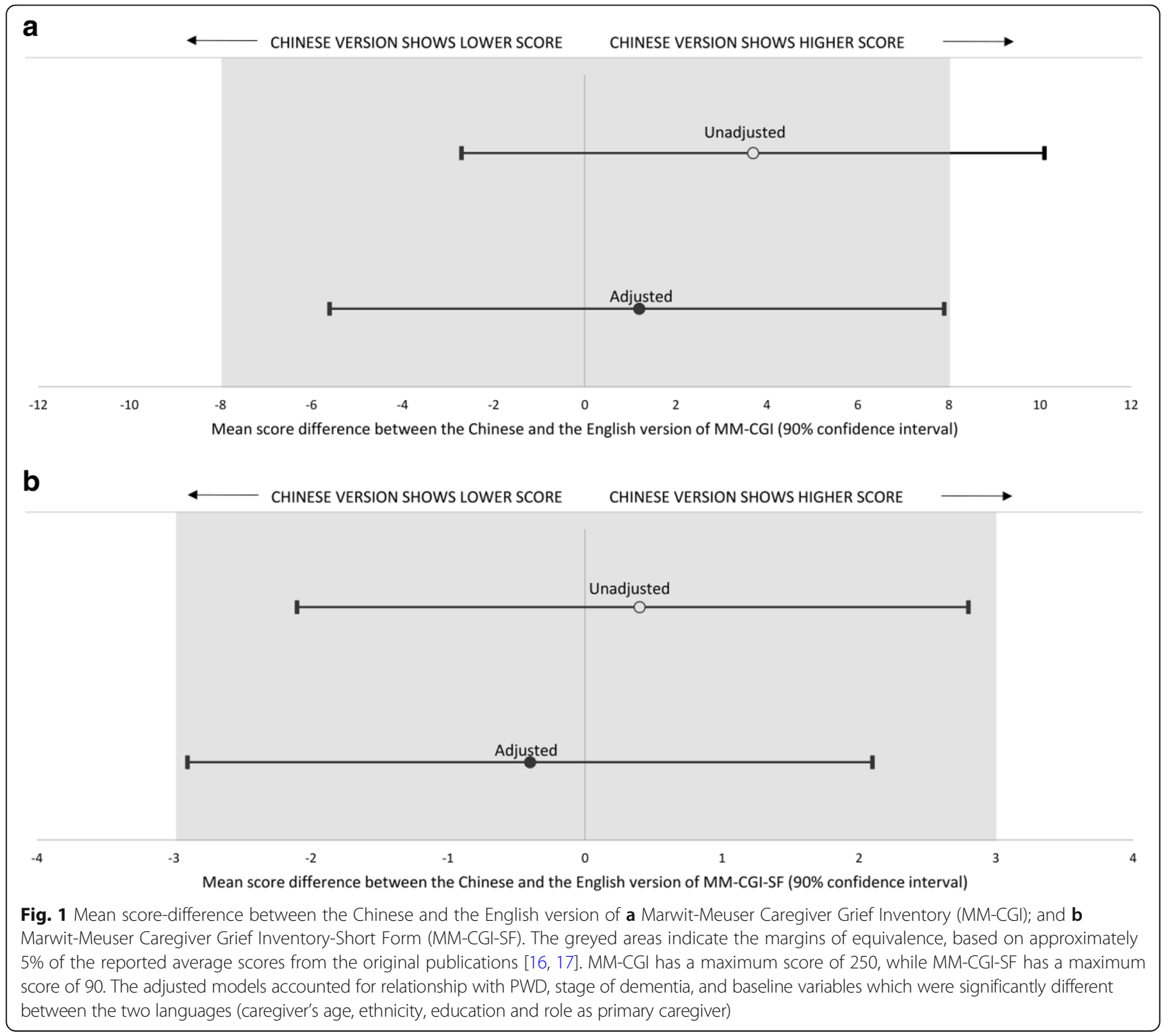


Table 2 Score-difference between the language versions of MM-CGI subscales and MM-CGI-SF subscales

\begin{tabular}{|c|c|c|c|c|c|c|}
\hline \multirow[t]{2}{*}{ Subscales of MM-CGI and MM-CGI-SF } & \multicolumn{2}{|c|}{ Mean scores (SD) } & \multirow[t]{2}{*}{ Equivalence margin (5\%) ${ }^{\mathrm{a}}$} & \multirow[t]{2}{*}{ Equivalence margin $(10 \%)^{\mathrm{b}}$} & \multicolumn{2}{|c|}{$\begin{array}{l}\text { Difference between Chinese and } \\
\text { English version }(90 \% \mathrm{Cl})\end{array}$} \\
\hline & Chinese & English & & & Unadjusted & Adjusted $^{c}$ \\
\hline \multicolumn{7}{|l|}{$\overline{\mathrm{MM}-\mathrm{CG}}$} \\
\hline Personal Sacrifice Burden subscale & $54.8(12.9)$ & $53.3(14.5)$ & \pm 3 & \pm 6 & $1.5(-1.2$ to 4.2$)$ & $1.0(-1.8$ to 3.8$)$ \\
\hline $\begin{array}{l}\text { Heartfelt Sadness and Longing } \\
\text { subscale }\end{array}$ & $44.0(8.8)$ & $43.3(11.9)$ & \pm 2 & \pm 4 & $0.8(-1.4$ to 2.9$)$ & $-0.5(-2.7$ to 1.8$)$ \\
\hline Worry and Felt Isolation subscale & $45.3(8.9)$ & $43.9(11.4)$ & \pm 2 & \pm 4 & $1.4(-0.6$ to 3.5$)$ & $0.7(-1.5$ to 2.9$)$ \\
\hline \multicolumn{7}{|l|}{ MM-CGI-SF } \\
\hline Personal Sacrifice Burden subscale & $18.7(4.7)$ & $18.2(5.3)$ & \pm 1 & \pm 2 & $0.4(-0.5$ to 1.4$)$ & $0.3(-0.7$ to 1.4$)$ \\
\hline $\begin{array}{l}\text { Heartfelt Sadness and Longing } \\
\text { subscale }\end{array}$ & $17.5(3.6)$ & $17.7(4.8)$ & \pm 1 & \pm 2 & $-0.2(-1.1$ to 0.6$)$ & $-0.6(-1.5$ to 0.3$)$ \\
\hline Worry and Felt Isolation subscale & $15.7(4.0)$ & $15.6(4.9)$ & \pm 1 & \pm 2 & $0.2(-0.7$ to 1.1$)$ & $-0.2(-1.1$ to 0.8$)$ \\
\hline
\end{tabular}

MM-CGI, Marwit-Meuser Caregiver Grief Inventory; MM-CGI-SF, Marwit-Meuser Caregiver Grief Inventory-Short Form; SD, standard deviation; Cl, confidence interval ${ }^{2}$ The pre-defined margins of equivalence represented approximately $5 \%$ of the reported average subscale scores from the original publications [16, 17] ${ }^{b}$ The pre-defined margins of equivalence represented approximately $10 \%$ of the reported average subscale scores from the original publications [16, 17] 'The adjusted models accounted for relationship with PWD, stage of dementia, and baseline variables which were significantly different between the two languages (caregiver's age, ethnicity, education and role as primary caregiver)

The Chinese and English versions showed similar mean scores: 144.1 (SD 28.0) and 140.5 (SD 35.6) respectively for MM-CGI; and 51.9 (SD 10.6) and 51.5 (SD 13.5) respectively for MM-CGI-SF. The mean scoredifference between the Chinese and English versions are illustrated in Fig. 1. In the adjusted model, the scoredifference was 1.2 (90\% CI -5.6 to 7.9) for MM-CGI and - 0.4 (90\% CI -2.9 to 2.1) for MM-CGI-SF. The 90\% CI for the adjusted score-differences fell within our predefined margins of equivalence $( \pm 8$ for MM-CGI and \pm 3 for MM-CGI-SF) and indicated equivalence of the scores. The adjusted score-differences for the subscales of MM-CGI and MM-CGI-SF are presented in Table 2. Although the subscales did not meet the 5\% equivalence margin, all of them had the $90 \% \mathrm{CI}$ falling within the less-stringent $10 \%$ margin which indicated possible equivalence.

The two language versions demonstrated similar patterns in internal consistency reliability, test-retest reliability, known-group validity and construct validity. The internal consistency and test-retest reliability of the two versions are presented in Table 3, with the $95 \%$ CI of all the reliability indices $\geq 0.70$. For known-group validity, both versions showed similar characteristics of higher MM-CGI scores in spouses and in later stage of dementia (see Fig. 2 for MM-CGI; and Fig. 3 for MM-CGI-SF). For construct validity (Table 4), both language versions correlated strongly (Spearman's $\rho>0.50$ ) [44] with the total scores of caregiver burden (ZBI) and depression (CES-D) $(\rho=0.61-0.77)$; and less strongly $(\rho \leq 0.50)$ [44] with Finance subscale of ZBI, Positive Affect subscale of CES-D and Interpersonal Problems subscale of CES-D $(\rho=0.16-0.49)$.

\section{Discussion}

This is the first study to produce the Mandarin-Chinese version of MM-CGI and MM-CGI-SF, and compare their psychometric properties to the original English version. It was possible to conduct such a study because Singapore is among the few countries in the world where both the Chinese and English languages are used as commonly. We demonstrated the equivalence of total scores between the Chinese and English versions, and also demonstrated possible equivalence (at $\pm 10 \%$ margins) in the subscale

Table 3 Comparison of the pattern in reliability indices between the Chinese and English versions of Marwit-Meuser Caregiver Grief Inventory

\begin{tabular}{llr}
\hline & Chinese version $(n=103)$ & English version $(n=291)$ \\
\hline Internal consistency reliability, Cronbach's a $(95 \% \text { Cl })^{\text {a }}$ & & $0.98(0.97-0.98)$ \\
MM-CGI & $0.96(0.95-0.97)$ & $0.94(0.93-0.95)$ \\
MM-CGI-SF & $0.91(0.87-0.94)$ & $0.90(0.86-0.93)$ \\
Test-retest reliability, ICC $(95 \%$ Cl) & & $0.88(0.84-0.92)$ \\
MM-CGI & $0.83(0.76-0.91)$ & $0.79(0.70-0.88)$ \\
MM-CGI-SF & & \\
\hline
\end{tabular}

MM-CGI Marwit-Meuser Caregiver Grief Inventory, MM-CGI-SF Marwit-Meuser Caregiver Grief Inventory-Short Form, Cl confidence interval, ICC intraclass correlation coefficient

${ }^{a}$ The $95 \% \mathrm{Cl}$ of the reliability indices were estimated using the bootstrap method 

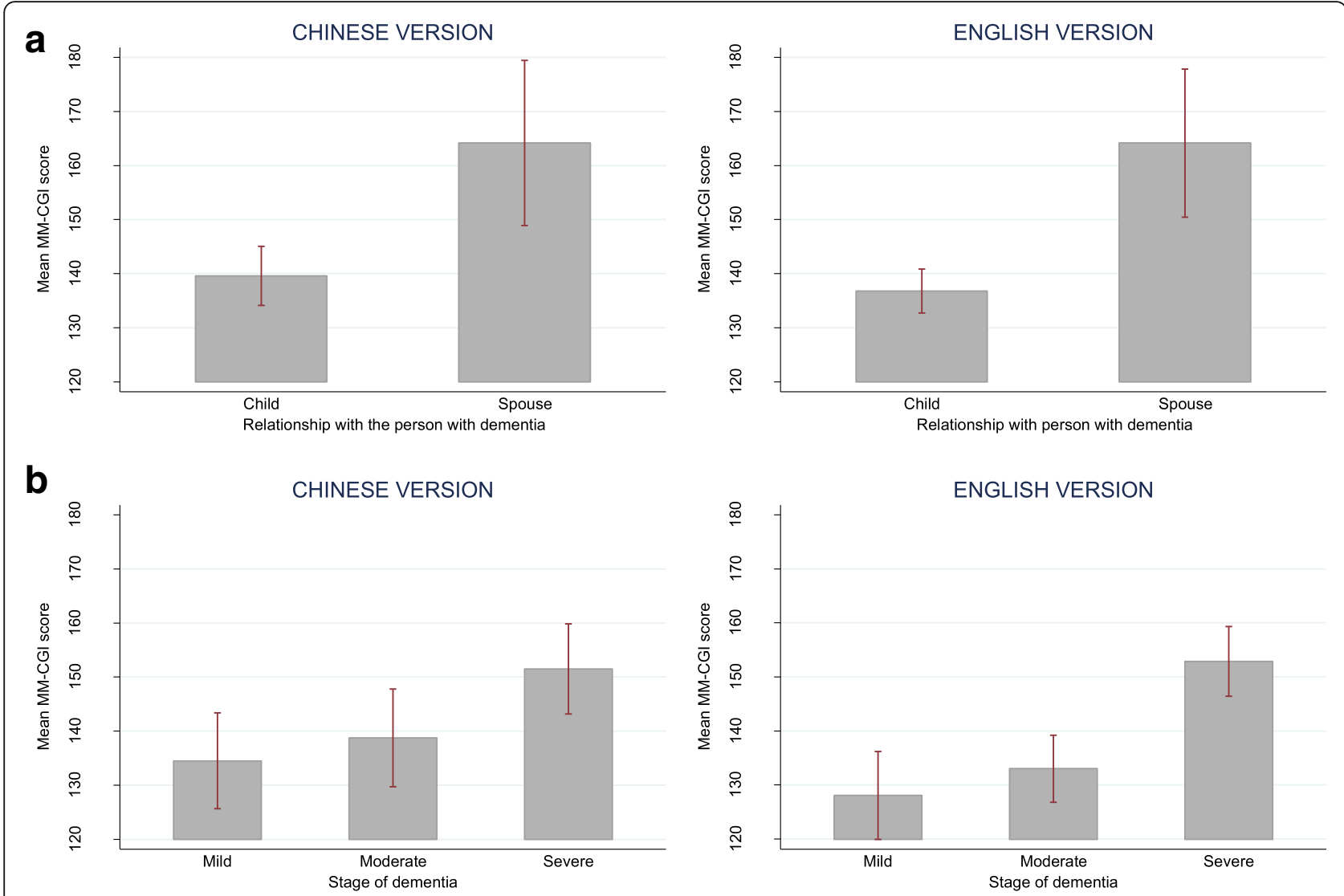

Fig. 2 Comparison of the mean scores of Marwit-Meuser Caregiver Grief Inventory (MM-CGI) between groups which are known to differ in pre-death grief level, in the assessment of known-group validity. Figure a shows the mean scores of MM-CGI for child and spousal caregivers. Figure $\mathbf{b}$ shows the mean scores of MM-CGl at various stages of dementia. The vertical lines of error bar indicate the $95 \%$ confidence interval of the scores

scores even though the sample size in the Chinese version $(n=103)$ was limited and may not have had sufficient power to establish equivalence using the more stringent $\pm 5 \%$ margins. Additionally, we showed that the Chinese and English versions performed similarly in terms of internal consistency reliability, test-retest reliability, knowngroup validity and construct validity.

The findings from this study have several implications. With the availability of the Mandarin-Chinese version of MM-CGI, we can now expand the reach of PDG detection to family caregivers who use Mandarin-Chinese as their primary language. This is noteworthy as the Mandarin-Chinese language is used by approximately one-sixth of the world's population [18], and Chinesespeaking populations have among the largest number of people living with dementia [1]. Detection of PDG provides an added dimension to supporting the emotional needs of family caregivers who are often the primary persons that provide most of the informal care to PWD [45] and thus can benefit from appropriate interventions for PDG.

The availability of the Mandarin-Chinese MM-CGI opens the way for further research to evaluate the efficacy of PDG interventions in dementia caregiving. To date, no intervention-studies have been conducted in the Chinese-speaking populations to address those with high PDG, possibly hindered by the lack of a relevant Chinese scale to assess PDG. Although prior studies have demonstrated the benefits of PDG interventions [7, 46, 47], they were primarily conducted in Caucasian populations. We remain uncertain whether PDG interventions are equally effective in the Chinese community, and whether there is a need to adapt the interventions to ensure that they can be culturally-relevant and sensitive to Chinese caregivers. In designing future intervention-studies for the Chinese community, researchers may possibly take reference from prior literature in this area [46-49] and include some of the commonly employed strategies such as: (1) encouraging caregivers to tell the story of the PWD through which they are helped to identify and process the painful emotions associated with the loss; (2) normalizing their emotions related to the loss and findings ways to remain connected with other caregivers; (3) educating caregivers on new ways to remain connected to the PWD such as through spiritual practices, celebrations, humor, life review and therapeutic touch; and (4) 


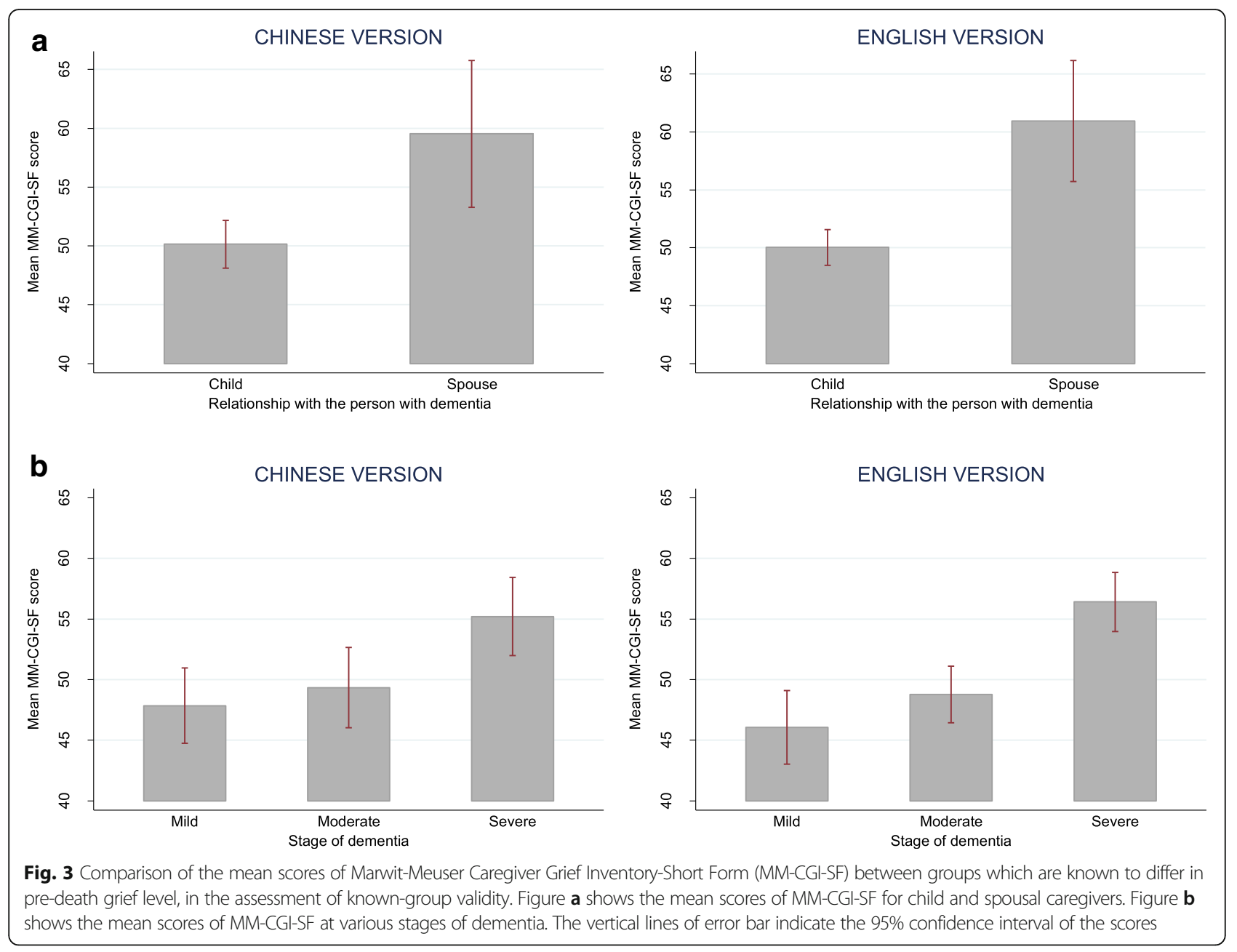

focusing on anticipatory grief and preparing the caregivers for potential future losses.

Measurement equivalence between the Chinese and English MM-CGI attests to the cross-cultural compatibility of the scale. Even though the Chinese and English languages are vastly different in their writing system (logogram- versus alphabet-based) and sentence structure, it was still possible to establish the measurement equivalence of the two versions. This supports the universal experience of PDG in caregivers of PWD [8, 38], and affirms the applicability of the PDG construct in the Chinese population. Indirectly, the measurement equivalence also provides evidence of the linguistic and cognitive equivalence between the Chinese and English versions [50]. It lends credence to the qualitative methods that were used to translate the Chinese version in this study.

Establishing measurement equivalence lays the ground for future research on the cross-cultural aspects of PDG in dementia caregiving. Although prior literature has suggested the influence of culture on the expression of grief $[21,38]$, we have limited understanding to date on how the characteristics of PDG vary across ethnic groups to affect dementia caregiving. It is now possible to use MM-CGI as a valid measure to compare PDG between the English- and Chinese-speaking populations because differences in PDG scores between the populations can be attributed to true variations in cultures per se, excluding scale language as the cause of differences. This is pertinent in the light of recent evidence demonstrating the influence of scale language on individuals' responses to culture-related questionnaires [20].

Finally, evidence of equivalence justifies the pooling of data from the two language versions to improve statistical power and representativeness of future research on PDG. This can be especially relevant in multi-ethnic populations such as ours where the use of vernacular prevails in different subgroups of the population. Using this study as an illustration, as seen in Table 1, the English version captured a subpopulation which was younger and more educated, while the Chinese version comprised those who were older and less educated. Combining data from both language versions is thereby more representative of the overall population of caregivers. 
Table 4 Comparison of correlation pattern among various scales, using Spearman's correlation coefficient $\rho(n=394)^{a}$

\begin{tabular}{|c|c|c|c|c|}
\hline & \multicolumn{2}{|l|}{ MM-CGI } & \multicolumn{2}{|c|}{ MM-CGI-SF } \\
\hline & Chinese & English & Chinese & English \\
\hline ZBI total scale & $0.76^{e}$ & $0.77^{\mathrm{e}}$ & $0.69^{e}$ & $0.73^{\mathrm{e}}$ \\
\hline Burden in the Relationship subscale & 0.72 & 0.69 & 0.68 & 0.68 \\
\hline Emotional Well-being subscale & 0.64 & 0.73 & 0.60 & 0.69 \\
\hline Social and Family Life subscale & 0.65 & 0.71 & 0.59 & 0.68 \\
\hline Loss of Control subscale & 0.64 & 0.68 & 0.55 & 0.63 \\
\hline Finances subscale & $0.35^{f}$ & $0.49^{f}$ & $0.30^{\mathrm{b}, \mathrm{f}}$ & $0.45^{f}$ \\
\hline CES-D total scale & $0.72^{\mathrm{e}}$ & $0.77^{\mathrm{e}}$ & $0.61^{\mathrm{e}}$ & $0.75^{\mathrm{e}}$ \\
\hline Depressed Affect subscale & 0.74 & 0.75 & 0.65 & 0.71 \\
\hline Somatic Symptoms subscale & 0.75 & 0.75 & 0.67 & 0.72 \\
\hline Interpersonal Problems subscale & $0.36^{f}$ & $0.48^{f}$ & $0.33^{f}$ & $0.47^{f}$ \\
\hline Positive Affect subscale & $-0.27^{c, f}$ & $-0.43^{f}$ & $-0.16^{\mathrm{d}, \mathrm{f}}$ & $-0.39^{f}$ \\
\hline
\end{tabular}

MM-CGI Marwit-Meuser Caregiver Grief Inventory, MM-CGI-SF Marwit-Meuser Caregiver Grief Inventory-Short Form, ZBI Zarit Burden Interview, CES-D Centre for Epidemiologic Studies Depression Scale

${ }^{\text {a All the unmarked correlations had } p<0.001}$

${ }^{\mathrm{b}} p=0.002$

${ }^{c} p=0.006$

${ }^{d} p=0.115$

eSimilar to the original studies [16, 17], MM-CGI and MM-CGI-SF correlated strongly ( $\rho>0.50)$ [44] with caregiver burden and depression scales because they measure related, though discriminable, phenomena in caregiving

${ }^{f} \mathrm{MM}-\mathrm{CGI}$ and MM-CGI-SF correlated less strongly $(\rho \leq 0.50)$ [44] with Finance subscale of ZBI, Positive Affect subscale of CES-D and Interpersonal Problems subscale of CES-D, because pre-death grief is expected to differ from constructs such as financial difficulties, positive feelings, or the feeling that others are being critical

Some limitations of the study should be noted. First, the participants were recruited from tertiary dementia services and may not fully represent those in the community. However, this is less likely a problem considering that most of the PWD in Singapore still receive their dementia care from tertiary centers, and the two recruitment centres in this study are the only two dementia services that serve the population in the North-East of Singapore. Second, the proportion of spousal caregivers in this study were much lower than that of the children caregivers (Table 1). Although it is possible that spousal caregivers may have been under-represented, this is less likely considering our proportion of spousal caregivers (13.7\%) were not much different from the $16.0 \%$ reported in a separate study based on nationally representative samples [51]. Third, we had a smaller sample size for participants who completed the Chinese version of MM-CGI $(n=103)$. At the point of recruitment, our participants were given the choice to complete either the Chinese MM-CGI (from the current study) or the English MM-CGI (from our separate study [8]). Only about one-third of our participants chose to complete the Chinese version of MM-CGI and these participants were more likely to be older and less educated. This is not inconsistent with what we would have expected of the population in Singapore [52] - the younger population is typically more educated and bilingual (competent in English and Chinese), and has a preference for the English language; while the older population is less educated and usually more competent in Chinese than English. However, the smaller sample size in the Chinese version $(n=103)$ resulted in some limitations in our analyses. Ideally, we would have evaluated the factor structure of Chinese MM-CGI. However, such analysis is less recommended when the sample size is around 100 or less because it may produce improper solutions and unstable parameter estimates [53]. Fourth, as the scales were self-administered, it is possible that caregivers with lower literacy were under-represented. Fifth, the stage of dementia was assessed only based on self-reports by family caregivers, which may lack the precision of other staging instruments that also encompass objective assessments by the healthcare professionals. Sixth, our definitions on the equivalence-margins required some subjectivity in deciding on the values that would be small enough to reasonably claim equivalence, as there were no previous studies to provide guidance. Regardless, we have stated the rationale for our choice of the equivalencemargins to allow readers to judge whether these values are reasonable. Last, the MM-CGI-SF in this study was administered as part of MM-CGI and it might have different properties had it been administered separately on its own.

\section{Conclusion}

In summary, this study has produced the MandarinChinese version of MM-CGI and demonstrated its measurement equivalence, as compared to the English version, in detecting PDG. It opens the way for PDG assessment and intervention in Chinese-speaking caregivers, as well as cross-cultural research on PDG in dementia caregiving. 


\section{Additional file}

Additional file 1: The final version of the Chinese Marwit-Meuser Caregiver Grief Inventory. (DOCX $1019 \mathrm{~kb}$ )

\section{Abbreviations}

CES-D: Centre for Epidemiologic Studies Depression Scale; Cl: confidence interval; DSM-5: the fifth edition of Diagnostic and Statistical Manual of Mental Disorders; DSM-III-R: the revised third edition of Diagnostic and Statistical Manual of Mental Disorders; ICC: intraclass correlation coefficient; ISPOR: the International Society for Pharmacoeconomics and Outcomes Research; MM-CGI: Marwit-Meuser Caregiver Grief Inventory; MM-CGI-SF: Marwit-Meuser Caregiver Grief Inventory-Short Form; PDG: Pre-death grief; PWD: Persons with dementia; SD: Standard deviation; ZBI: Zarit Burden Interview

\section{Acknowledgements}

The authors thank the following persons for their involvement in the translation process of the Chinese scale: Sanni June Chyn Leong, Beow Im Yeap, Hwei Lee Soh, Derina Li-Ling Chong, Kheng Siew Tan, Yanli Hou, Haiyan Li and Chunjuan Lu.

\section{Funding}

This research was supported by the Singapore Ministry of Health's National Medical Research Council under the Centre Grant Program (Grant No.: NMRC/ CG/004/2013). It also received pilot funding from the National University of Singapore. Separately, the first author (TML) was supported by research grants under the Singapore Ministry of Health's National Medical Research Council (Grant No.: NMRC/Fellowship/0030/2016 and NMRC/CSSSP/0014/2017). The funding sources had no involvement in any part of the project.

\section{Availability of data and materials}

The datasets used and/or analysed during the current study are available from the corresponding author on reasonable request.

\section{Authors' contributions}

All authors have read and approved the manuscript. Study concept and design: TML. Acquisition of data: TML, PY, SBH. Analysis of data: TML, BCT. Interpretation of data: TML, PY, NL, GCHK, BCT. Drafting of the manuscript: TML. Revision of the manuscript: TML, PY, NL, SBH, GCHK, BCT. Funding: TML. Project supervision: TML.

\section{Ethics approval and consent to participate}

The Domain Specific Review Board of Singapore granted ethical approval for the study. Written informed consent was obtained from all the participants.

\section{Competing interests}

The authors declare that they have no competing interests.

\section{Publisher's Note}

Springer Nature remains neutral with regard to jurisdictional claims in published maps and institutional affiliations.

\section{Author details}

'Department of Geriatric Psychiatry, Institute of Mental Health, 10 Buangkok View, Singapore, Singapore. ${ }^{2}$ Psychotherapy Service, Institute of Mental Health, Singapore, Singapore. ${ }^{3}$ Saw Swee Hock School of Public Health, National University of Singapore, Singapore, Singapore. ${ }^{4}$ Department of Geriatric Medicine, Khoo Teck Puat Hospital, Singapore, Singapore. ${ }^{5}$ Geriatric Education and Research Institute, Singapore, Singapore.

Received: 27 September 2017 Accepted: 1 May 2018

Published online: 11 May 2018

\section{References}

1. Prince MJ, Wimo A, Guerchet MM, Ali GC, Wu Y-T, Prina M: World Alzheimer report 2015 - the global impact of dementia: Alzheimer's disease international; 2015

2. Mannion E. Alzheimer's disease: the psychological and physical effects of the caregiver's role. Part 1. Nurs Older People. 2008;20(4):27-32.
3. Gilhooly KJ, Gilhooly MLM, Sullivan MP, Mclntyre A, Wilson L, Harding E, Woodbridge R, Crutch S. A meta-review of stress, coping and interventions in dementia and dementia caregiving. BMC Geriatr. 2016;16(1):106.

4. Vitaliano PP, Zhang J, Scanlan JM. Is caregiving hazardous to one's physical health? A meta-analysis. Psychol Bull. 2003;129(6):946-72.

5. Toot S, Swinson T, Devine M, Challis D, Orrell M. Causes of nursing home placement for older people with dementia: a systematic review and metaanalysis. Int Psychogeriatr. 2016;29(2):195-208.

6. Lwi SJ, Ford BQ, Casey JJ, Miller BL, Levenson RW. Poor caregiver mental health predicts mortality of patients with neurodegenerative disease. Proc Natl Acad Sci U S A. 2017;114(28):7319-24.

7. MacCourt P, McLennan M, Somers S, Krawczyk M. Effectiveness of a grief intervention for caregivers of people with dementia. Omega (Westport). 2017:75(3):230-47.

8. Liew TM, Yeap BI, Koh GC, Gandhi M, Tan KS, Luo N, Yap P. Detecting Predeath grief in family caregivers of persons with dementia: validity and utility of the Marwit-Meuser caregiver grief inventory in a multiethnic Asian population. Gerontologist. 2018;58(2):e150-9.

9. Feast A, Orrell M, Charlesworth G, Melunsky N, Poland F, Moniz-Cook E. Behavioural and psychological symptoms in dementia and the challenges for family carers: systematic review. Br J Psychiatry. 2016;208(5):429-34.

10. Pozzebon M, Douglas J, Ames D. Spouses' experience of living with a partner diagnosed with a dementia: a synthesis of the qualitative research. Int Psychogeriatr. 2016;28(4):537-56.

11. Chan D, Livingston $G$, Jones L, Sampson EL. Grief reactions in dementia carers: a systematic review. Int J Geriatr Psychiatry. 2013;28(1):1-17.

12. Lindauer $A$, Harvath TA. Pre-death grief in the context of dementia caregiving: a concept analysis. J Adv Nurs. 2014;70(10):2196-207.

13. Piiparinen $\mathrm{R}$, Whitlatch CJ. Existential loss as a determinant to wellbeing in the dementia caregiving dyad: a conceptual model. Dementia. 2011;10(2):185-201.

14. Holley CK, Mast BT. The impact of anticipatory grief on caregiver burden in dementia caregivers. Gerontologist. 2009;49(3):388-96.

15. Walker RJ, Pomeroy EC. The impact of anticipatory grief on caregivers of persons with Alzheimer's disease. Home Health Care Serv Q. 1997; 16(1-2):55-76.

16. Marwit SJ, Meuser TM. Development and initial validation of an inventory to assess grief in caregivers of persons with Alzheimer's disease. Gerontologist. 2002;42(6):751-65.

17. Marwit SJ, Meuser TM. Development of a short form inventory to assess grief in caregivers of dementia patients. Death Stud. 2005;29(3):191-205.

18. Paul LM, Gary F, Simons GF. Fennig CD (eds.): Ethnologue: languages of the world, nineteenth edition. Dallas, Texas: SIL International; 2016.

19. Chan WCH, Wong B, Kwok T, Ho F. Assessing grief of family caregivers of people with dementia: validation of the Chinese version of the MarwitMeuser caregiver grief inventory. Health Soc Work. 2017:42(3):151-8.

20. Harzing AW. Does the use of English-language questionnaires in crossnational research obscure national differences. Int J Cross Cult Manage. 2005;5(2):213-24.

21. Eisenbruch M. Cross-cultural aspects of bereavement. II: ethnic and cultural variations in the development of bereavement practices. Cult Med Psychiatry. 1984;8(4):315-47.

22. Wild D, Grove A, Martin M, Eremenco S, McElroy S, Verjee-Lorenz A, Erikson P. Principles of good practice for the translation and cultural adaptation process for patient-reported outcomes (PRO) measures: report of the ISPOR task force for translation and cultural adaptation. Value Health. 2005:8(2):94-104.

23. Pearlin LI, Mullan JT, Semple SJ, Skaff MM. Caregiving and the stress process: an overview of concepts and their measures. Gerontologist. 1990;30(5):583-94.

24. Dempsey M, Baago S. Latent grief: the unique and hidden grief of carers of loved ones with dementia. Am J Alzheimers Dis Other Demen. 1998;13(2):84-91.

25. Zarit SH, Zarit JM. Instructions for the burden interview. University Park, PA: Pennsylvania State University; 1987

26. Yu J, Yap P, Liew TM. The optimal short version of the Zarit burden interview for dementia caregivers: diagnostic utility and externally validated cutoffs. Aging Ment Health. 2018:1-5.

27. Rankin ED, Haut MW, Keefover RW, Franzen MD. The establishment of clinical cutoffs in measuring caregiver burden in dementia. Gerontologist. 1994;34(6):828-32

28. Radloff LS. The CES-D scale - a self-report depression scale for research in the general population. Appl Psych Meas. 1977;1(3):385-401. 
29. Vilagut G, Forero CG, Barbaglia G, Alonso J. Screening for depression in the general population with the Center for Epidemiologic Studies Depression (CES-D): a systematic review with meta-analysis. PLoS One. 2016;11(5):e0155431.

30. Seng BK, Luo N, Ng WY, Lim J, Chionh HL, Goh J, Yap P. Validity and reliability of the Zarit burden interview in assessing caregiving burden. Ann Acad Med Singap. 2010;39(10):758-63.

31. Stahl D, Sum CF, Lum SS, Liow PH, Chan YH, Verma S, Chua HC, Chong SA. Screening for depressive symptoms: validation of the center for epidemiologic studies depression scale (CES-D) in a multiethnic group of patients with diabetes in Singapore. Diabetes Care. 2008;31(6):1118-9.

32. American Psychiatric Association. Diagnostic and statistical manual of mental disorders: DSM-III-R. Washington: American Psychiatric Association; 1987.

33. Forsell Y, Fratiglioni L, Grut M, Viitanen M, Winblad B. Clinical staging of dementia in a population survey: comparison of DSM-III-R and the Washington University clinical dementia rating scale. Acta Psychiatr Scand. 1992;86(1):49-54.

34. Juva K, Sulkava R, Erkinjuntti T, Ylikoski R, Valvanne J, Tilvis R. Staging the severity of dementia: comparison of clinical (CDR, DSM-III-R), functional (ADL, IADL) and cognitive (MMSE) scales. Acta Neurol Scand. 1994;90(4):293-8.

35. Rikkert MG, Tona KD, Janssen L, Burns A, Lobo A, Robert P, Sartorius N, Stoppe G, Waldemar G. Validity, reliability, and feasibility of clinical staging scales in dementia: a systematic review. Am J Alzheimers Dis Other Demen. 2011;26(5):357-65.

36. Morris JC. The clinical dementia rating (CDR): current version and scoring rules. Neurology. 1993:43(11):2412-4.

37. American Psychiatric Association. Diagnostic and statistical manual of mental disorders: DSM-5. Washington, DC: Amer Psychiatric Pub Incorporated; 2013.

38. Liew TM. Applicability of the pre-death grief concept to dementia family caregivers in Asia. Int J Geriatr Psychiatry. 2016;31(7):749-54.

39. Machin D, Campbell MJ, Tan SB, Tan SH. Sample size tables for clinical studies. West Sussex: Wiley; 2009.

40. Wang D, Bakhai A. Clinical trials: a practical guide to design, analysis, and reporting. London: Remedica; 2006.

41. Schuirmann DJ. A comparison of the two one-sided tests procedure and the power approach for assessing the equivalence of average bioavailability. J Pharmacokinet Biopharm. 1987;15(6):657-80.

42. Nunnally JC. Psychometric theory, 3rd ed. edn. New York: McGraw-Hill; 1994.

43. Fayers PM. Quality of life the assessment, analysis and interpretation of patient-reported outcomes, 2nd ed. edn. Chichester, England: Wiley; 2007.

44. Juniper EF, Guyatt GH, Jaeschke R: How to develop and validate a new healthrelated quality of life instrument. In: Quality of Life and Pharmacoeconomics in Clinical Trials. Edited by B S: Philadelphia: Lippincott-Raven; 1996.

45. World Health Organization. Dementia: a public health priority. United Kingdom: World Health Organization; 2012.

46. Ott CH, Kelber ST, Blaylock M. "Easing the way" for spouse caregivers of individuals with dementia: a pilot feasibility study of a grief intervention. Res Gerontol Nurs. 2010;3(2):89-99.

47. Meichsner F, Wilz G. Dementia caregivers' coping with pre-death grief: effects of a CBT-based intervention. Aging Ment Health. 2016:1-8.

48. Meichsner F, Schinkothe D, Wilz G. Managing loss and change: grief interventions for dementia caregivers in a CBT-based trial. Am J Alzheimers Dis Other Demen. 2016;31(3):231-40.

49. Sanders S, Marwit SJ, Meuser TM, Harrington P. Caregiver grief in end-stage dementia:using the Marwit and Meuser caregiver grief inventory for assessment and intervention in social work practice. Soc Work Health Care. 2007:46(1):47-65.

50. Wild D, Eremenco S, Mear I, Martin M, Houchin C, Gawlicki M, Hareendran A, Wiklund I, Chong LY, von Maltzahn R, et al. Multinational trialsrecommendations on the translations required, approaches to using the same language in different countries, and the approaches to support pooling the data: the ISPOR patient-reported outcomes translation and linguistic validation good research practices task force report. Value Health. 2009;12(4):430-40.

51. Malhotra R, Chan A, Malhotra C, Ostbye T. Validity and reliability of the caregiver reaction assessment scale among primary informal caregivers for older persons in Singapore. Aging Ment Health. 2012;16(8):1004-15.

52. Singapore Department of Statistics: Census of population 2010. Republic of Singapore: Department of Statistics, Ministry of Trade \& Industry; 2011.

53. Marsh HW, Hau KT, Balla JR, Grayson D. Is more ever too much? The number of indicators per factor in confirmatory factor analysis. Multivariate Behav Res. 1998;33(2):181-220.

\section{Ready to submit your research? Choose BMC and benefit from:}

- fast, convenient online submission

- thorough peer review by experienced researchers in your field

- rapid publication on acceptance

- support for research data, including large and complex data types

- gold Open Access which fosters wider collaboration and increased citations

- maximum visibility for your research: over $100 \mathrm{M}$ website views per year

At BMC, research is always in progress.

Learn more biomedcentral.com/submissions 